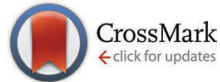

Cite this: Chem. Commun., 2016, 52,6185

Received 5th February 2016 Accepted 6th April 2016

DOI: $10.1039 / \mathrm{c} 6 \mathrm{cc} 01166 \mathrm{~g}$

www.rsc.org/chemcomm

\section{Tuning of photocatalytic reduction by conduction band engineering of semiconductor quantum dots with experimental evaluation of the band edge potential $\dagger$}

\author{
Takafumi Suzuki, ${ }^{a}$ Hiroto Watanabe, ${ }^{\mathrm{b}}$ Yuya Oaki ${ }^{\mathrm{a}}$ and Hiroaki Imai*a
}

\begin{abstract}
The conduction band edge (CBE) of $\mathrm{WO}_{3}$ quantum dots (QDs) was determined experimentally and engineered by size control to around one nanometer. Photocatalytic reactions of proton and molecular oxygen were tuned by changing the $\mathrm{CBE}$ of $\mathrm{WO}_{3}-\mathrm{QDs}$.
\end{abstract}

Photocatalytic reactions on semiconductor materials have been attracting much attention as an approach to solving problems of global energy and environmental issues by converting solar energy into chemical energy. ${ }^{1}$ Photocatalytic water splitting is being actively investigated to produce hydrogen as a future fuel. ${ }^{2}$ Photocatalytic decomposition of organic pollutants and photoreduction of carbon dioxide are examined for their potential for leading to a clean environment and as a solution to global warming, respectively. ${ }^{3,4}$ In photocatalytic reactions on semiconductor materials, photoexcited electrons in the conduction band and holes in the valence band cause reduction and oxidation reactions, respectively. ${ }^{5,6}$ The generation of hydrogen, the production of superoxide radicals, and the decomposition of carbon dioxide are induced by photoexcited electrons in the conduction band. Therefore, a detailed investigation of the reduction reactions induced by photoexcited electrons is required to promote efficient light energy harvesting.

Photocatalysts reduce chemical species when their conduction band edge (CBE) potential is more negative than the reduction potential of the reactants. Fujishima et al. reported the photocatalytic reduction of carbon dioxide to methanol $\left(\mathrm{CH}_{3} \mathrm{OH}\right)$ on various semiconductor photocatalysts suspended in water. ${ }^{7}$ The production yields increased as the $\mathrm{CBE}$ potential became more negative than the redox potential of $\mathrm{H}_{2} \mathrm{CO}_{3} / \mathrm{CH}_{3} \mathrm{OH}$. Recently, the architecture of the advanced photocatalysts has been studied by using solid solutions. We can control the band structure of solid

\footnotetext{
${ }^{a}$ Department of Applied Chemistry, Faculty of Science and Technology,

Keio University, 3-14-1 Hiyoshi, Kohoku-ku, Yokohama 223-8522, Japan. E-mail: hiroaki@applc.keio.ac.jp

${ }^{b}$ Tokyo Metropolitan Industrial Technology Research Institute,

2-4-10 Aomi. Koto-ku, Tokyo 135-0064, Japan

$\dagger$ Electronic supplementary information (ESI) available. See DOI: 10.1039/ c6cc01166g
}

solution photocatalysts by changing their composition. Tsuji et al. reported efficient photocatalytic hydrogen production using $(\mathrm{AgIn})_{x} \mathrm{Zn}_{2(1-x)} \mathrm{S}_{2}$ photocatalysts with a tunable bandgap. ${ }^{8}$ Photocatalytic activity increased with a CBE potential that was sufficiently negative to reduce $\mathrm{H}_{2} \mathrm{O}$ to $\mathrm{H}_{2}$ as the ratio of $\mathrm{ZnS}$ increased. Liu et al. investigated the correlation between band structures and the photocatalytic activities of $\mathrm{Cd}_{x} \mathrm{Cu}_{y} \mathrm{Zn}_{1-x-y} \mathrm{~S}$ solid solutions. ${ }^{9}$ $\mathrm{Cd}_{x} \mathrm{Cu}_{y} \mathrm{Zn}_{1-x-y} \mathrm{~S}$ with a lower $x$ value has a higher CBE. The quantum efficiency of the photocatalysts for $\mathrm{H}_{2}$ production was enhanced as the CBE of the solid solution shifted upon changing the composition. However, the correlation between the CBE and the photocatalytic activity cannot be investigated in detail because of the different overvoltages caused by the change of substance and composition. The variation of the reduction efficiency by tuning its band structure should be investigated using a single semiconductor without changing its composition. Thus, we can evaluate the CBE of the semiconductor experimentally for a detailed discussion on the photocatalytic performance.

Quantum dots (QDs) are tiny particles of semiconductor materials with a size below $10 \mathrm{~nm} \cdot{ }^{10,11}$ Tuning the CBE of a single semiconductor is achieved through the quantum size effect by changing the size of the QDs. Since typical QDs of CdSe and ZnS are susceptible to oxidation, metal oxide QDs are required for practical photocatalytic systems. However, the quantum size effect on the metal oxide QDs is generally weak in the size region larger than $2 \mathrm{~nm}$ due to their small Bohr exciton radius. ${ }^{12}$ In our previous study, we observed the remarkable expansion of the bandgap of $\mathrm{WO}_{3}$-QDs using the pores of supermicroporous silicas (SMPSs) as templates. ${ }^{13}$ The preparation of QDs smaller than $2 \mathrm{~nm}$ using SMPSs is beneficial because the bandgap of most metal oxides markedly expand in the size region. The photoreduction of molecular oxygen that does not occur with bulk $\mathrm{WO}_{3}$ was achieved by the bandgap expansion of $\mathrm{WO}_{3}$-QDs. However, the upshift of the CBE potential, which is essential for photoreduction, was not estimated experimentally and the correlation between the CBE and photoreduction activity was not examined in the previous work. The correlation between the $\mathrm{CBE}$ of $\mathrm{WO}_{3}$-QDs and the 
photoreduction reaction efficiency has not been investigated in detail because of a lack of experimental evaluation of the band edge potential.

In the present study, the size of $\mathrm{WO}_{3}$-QDs was strictly tuned to be below $1.8 \mathrm{~nm}$ by changing the pore size of SMPSs as templates and the amount of the precursor solution of $\mathrm{WO}_{3}$-QDs impregnated into SMPSs. The variation of the CBE of $\mathrm{WO}_{3}$-QDs was experimentally examined in combination with a photosensitive organic compound. Finally, we investigated the reactivity of the photocatalytic reduction as a function of the CBE of a single oxide semiconductor. We observed remarkably enhanced photoreduction of proton and molecular oxygen by the upshift of the $\mathrm{CBE}$ of $\mathrm{WO}_{3}$-QDs. The band engineering using the quantum size effect is found to be useful for tuning the efficiency of specific photocatalytic reactions.

$\mathrm{WO}_{3}$-QDs were prepared in the pores of SMPSs. The size of the $\mathrm{WO}_{3}$-QDs was controlled by changing the pore size of the SMPSs and the amount of the precursor solution of the $\mathrm{WO}_{3}$-QDs impregnated into the SMPSs (Fig. $\mathrm{S} 1$ and $\mathrm{S} 2$ in the ESI $\dagger$ ). The presence of the tungsten(vi) species in the SMPSs was confirmed by EDX and XPS (Fig. S3 and S4, ESI $\dagger$ ). The influence of surface defects (i.e. $\mathrm{W}^{5+}$ ) was negligible because of the absence of defect absorption in the UV-Vis spectra (Fig. S5, ESI $\dagger$ ). As shown in Fig. 1a-d, FE-TEM images indicate that the $\mathrm{WO}_{3}$-QDs were well dispersed in the SMPSs. $\mathrm{WO}_{3}$-QDs with a narrow size distribution were obtained by using the pores of SMPSs as templates. The size of the $\mathrm{WO}_{3}$-QDs clearly increased with the increased pore size of the SMPSs and the amount of the precursor solution of the $\mathrm{WO}_{3}$-QDs impregnated into the SMPSs (Fig. S6 and S7, ESI $\dagger$ ). We succeeded in controlling the size of the $\mathrm{WO}_{3}$-QDs in the range of 0.66 and $1.79 \mathrm{~nm}$ by changing the pore size of the SMPSs and the amount of the impregnated precursor solution.

The bandgap of $\mathrm{WO}_{3}$-QDs depended on their size due to the quantum size effect. Fig. S8 (ESI $\dagger$ ) shows Tauc plots of $\mathrm{C} n \times m$ whose diameters were directly estimated from FE-TEM images (Fig. 1a-d and Fig. S6, ESI $\dagger$ ). Because the smaller $\mathrm{WO}_{3}$-QDs show larger blue shifts of the absorption edge, the bandgap expands from 2.87 to $3.45 \mathrm{eV}$ by decreasing their size (Fig. 1e). The variation of the bandgap energy of $\mathrm{WO}_{3}$-QDs larger than $1.3 \mathrm{~nm}$ can be fitted using the Brus equation (Fig. 1e). ${ }^{14}$ The reduced mass of the electron and hole was calculated using the relation between the size of the $\mathrm{WO}_{3}$-QDs and the bandgap energy (Fig. S9, ESI $\dagger$ ). However, the bandgap energy for $\mathrm{WO}_{3}$-QDs smaller than $1.1 \mathrm{~nm}$ is overestimated by the Brus equation. Lippens et al. reported that the experimental values of the bandgap of CdS-QDs were lower than the theoretical values as their size decreased to below $3 \mathrm{~nm} .{ }^{15}$ The quantum size effect is relatively weakened in the small size region. Thus, a higher bandgap than $3.3 \mathrm{eV}$ can be obtained by miniaturizing of $\mathrm{WO}_{3}$ to $\sim 1 \mathrm{~nm}$.

We prepared $\mathrm{WO}_{3}$-QDs/phenol complexes to evaluate the $\mathrm{CBE}$ of $\mathrm{WO}_{3}$-QDs experimentally. Derivatives of phenol and catechol on metal oxide give photoinduced charge-transfer excitation (CTE) from their HOMO to the CBE of the metal oxide. ${ }^{16}$ We ignored the HOMO level pinning of phenol because
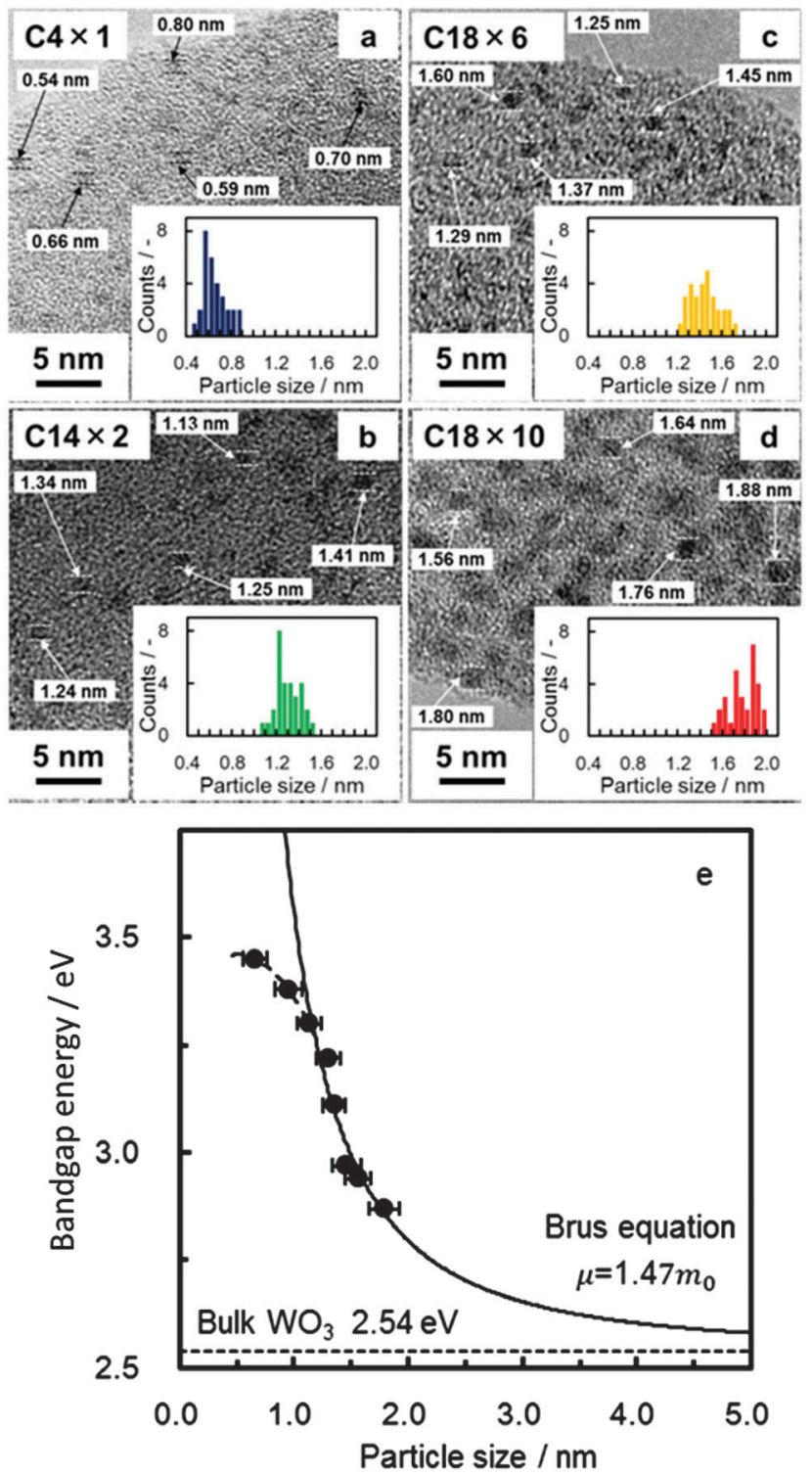

Fig. $1(a-d)$ Typical FE-TEM images of $\mathrm{Cn} \times m$ with histograms of the particle size of $\mathrm{WO}_{3}-\mathrm{QDs}$ and (e) the relationship between the particle size and the bandgap energy. The well-dispersed black dots are $\mathrm{WO}_{3}-\mathrm{QDs}$. The gray matrixes are SMPSs. The products are denoted as $\mathrm{C} n \times m(n=4-18$, $n$ : carbon number of the alkyl chain of $\operatorname{CnTAC}(B)$ which is related to the pore size of the SMPSs; $m=1-10, m$ : the amount of the precursor solution of the $\mathrm{WO}_{3}$-QDs impregnated into the SMPSs).

the HOMO of phenol is lower than the fermi energy of $\mathrm{WO}_{3}$-QDs. ${ }^{17}$ Therefore, we estimated the $\mathrm{CBE}$ of $\mathrm{WO}_{3}$-QDs by the surface complex formed with phenol. The color of $\mathrm{C} n \times m$ changed from white and yellow to orange and brown upon complexation of phenol. The dashed lines in Fig. 2a and Fig. S10 (ESI $\dagger$ ) are Tauc plots of $\mathrm{C} n \times m /$ phenol complexes. The red shift of the absorption edge was induced by the complexation of phenol.

The charge-transfer excitation energy $E_{\mathrm{CT}}$ was estimated from Tauc plots of $\mathrm{C} n \times m /$ phenol complexes. The HOMO of the phenol was evaluated to be $1.73 \mathrm{~V}_{\mathrm{SHE}}$ by photoemission yield spectroscopy in air (PYSA) (Fig. S11, ESI $\dagger$ ). 

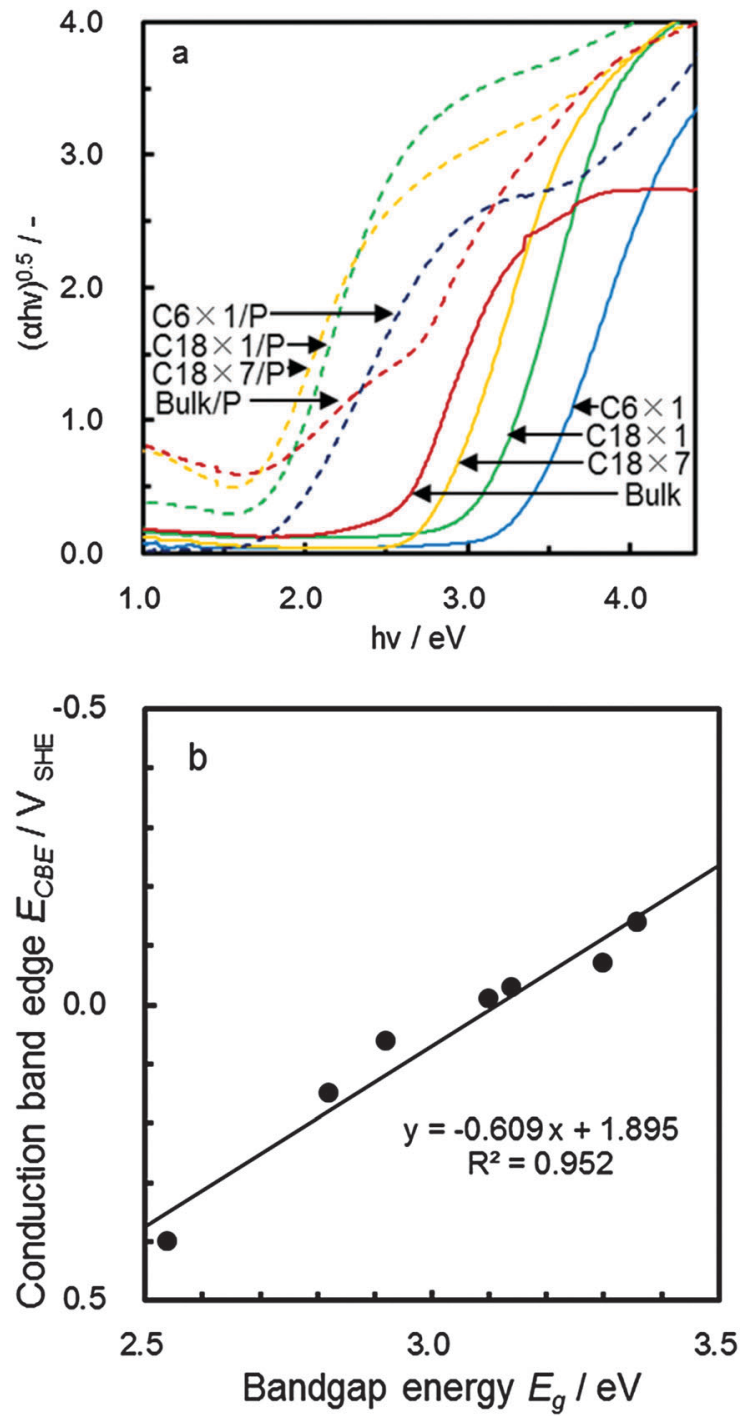

Fig. 2 (a) Tauc plots of $\mathrm{C} n \times m$ (solid lines) and $\mathrm{C} n \times m /$ phenol complexes ( $\mathrm{C} n \times m / \mathrm{P}$, dashed lines) and (b) the relation between the estimated CBE of $\mathrm{WO}_{3}-\mathrm{QDs}$ and the bandgap energy of $\mathrm{WO}_{3}-\mathrm{QDs}$.

We obtained the $\mathrm{CBE}$ of $\mathrm{WO}_{3}$-QDs using the following equation (Fig. S12, ESI $\dagger$ ).

$$
E_{\mathrm{CBE}}=E_{\mathrm{HOMO}(\text { phenol })}-E_{\mathrm{CT}}
$$

Fig. $2 \mathrm{~b}$ shows the relationship between the CBE estimated by the absorption of $\mathrm{WO}_{3}$-QDs/phenol complexes and the bandgap energy of $\mathrm{WO}_{3}$-QDs. The slope of the approximation of experimental CBE values (Fig. 2b, solid line) is given by the following equation:

$$
\text { Slope }=-\frac{\Delta E_{\mathrm{CBE}}}{\Delta E_{\mathrm{g}}}=-\frac{1 / m_{\mathrm{e}}}{1 / m_{\mathrm{e}}+1 / m_{\mathrm{h}}}=-\frac{m_{\mathrm{h}}}{m_{\mathrm{e}}+m_{\mathrm{h}}}
$$

The slope value -0.6 indicates that the effective mass of electron $m_{\mathrm{e}}$ is smaller than the effective mass of hole $m_{\mathrm{h}}$ in $\mathrm{WO}_{3}$. From the slope value and $\mu=1.47 m_{0}, m_{\mathrm{e}}$ and $m_{\mathrm{h}}$ are calculated to be $2.39 m_{0}$ and $3.83 m_{0}$, respectively. Therefore, the upshift of the CBE is larger than the downshift of the valence band edge (Fig. S12, ESI $\uparrow$ ).

Photoreduction of proton and molecular oxygen was performed under UV irradiation $(290-350 \mathrm{~nm})$ in the presence of ethanol as the sacrificial reagent. Equal amounts of $\mathrm{WO}_{3}$ were used for all photocatalytic activity evaluations (Tables S1 and S2, ESI $\dagger$ ). We synthesized the $\mathrm{WO}_{3}$-QDs with CBE between $+0.18 \mathrm{~V}_{\mathrm{SHE}}$ to $-0.10 \mathrm{~V}_{\mathrm{SHE}}$ in the same sized pores of SMPSs (pore size: $3.0 \mathrm{~nm}$ ), by changing the amount of the impregnated precursor solution. Therefore, the influence of the pore size of SMPSs on the photocatalytic efficiency was excluded.

The photoreduction efficiency of molecular oxygen was monitored by using the ESR spin trapping method in ethanol solution of 5,5-dimethyl-1-pyrroline- $N$-oxide (DMPO). The photogenerated $\mathrm{O}_{2}$ anion radicals were trapped by DMPO. We regarded the double integral values of the ESR signal of $\mathrm{DMPO}-\mathrm{OOH}$ as the efficiency of the photoreduction of molecular oxygen (Fig. S14, ESI $\dagger$ ). ${ }^{18}$ Closed circles in Fig. 3a show the relationship between the double integral ESR values and the $\mathrm{CBE}$ of $\mathrm{WO}_{3}$-QDs. The radicals were hardly produced by $\mathrm{WO}_{3}$ having $\mathrm{CBE}$ that was positive or less negative than the single-electron reduction potential of molecular oxygen $\left[E^{0}\left(\mathrm{HO}_{2} \cdot \mathrm{O}_{2}\right)=-0.05 \mathrm{~V}_{\mathrm{SHE}}\right]{ }^{19}$ On the other hand, photoreduction of molecular oxygen occurred when the $\mathrm{CBE}$ of $\mathrm{WO}_{3}$-QDs was more negative than the reduction potential. Efficiency was drastically boosted as the negative shift of the CBE from the reduction potential increased.

The photoreduction of proton was conducted in the aqueous suspension of the $\mathrm{WO}_{3}$-QDs in the presence of $20 \mathrm{vol} \%$ ethanol. The amounts of hydrogen molecules produced were monitored by gas chromatography. The open circles in Fig. 3a show the relationship between the efficiency of photoreduction of proton and the $\mathrm{CBE}$ potential of $\mathrm{WO}_{3}$-QDs. Photocatalytic production of hydrogen molecules was hardly observed with the $\mathrm{WO}_{3}$-QDs having a CBE potential that was more positive than $+0.1 \mathrm{~V}_{\mathrm{SHE}}$. On the other hand, photoreduction of proton occurred when the $\mathrm{CBE}$ potential of $\mathrm{WO}_{3}$-QDs was close to the reduction potential of proton $\left[E^{0}\left(\mathrm{H}_{2} / \mathrm{H}^{+}\right)=0 \mathrm{~V}_{\mathrm{SHE}}\right]$. The efficiencies were increased by the negative shift of the CBE. However, a decrease in the efficiency was observed when the CBE potential was more negative than $-0.05 \mathrm{~V}_{\mathrm{SHE}}$, which corresponds to the reduction potential of molecular oxygen. This decrease in the reduction efficiency was owing to the competition of the two reactions, i.e. reduction of molecular oxygen and proton. This suggests that the photoreduction of molecular oxygen preferentially occurred instead of hydrogen molecule evolution in the region of the CBE potential more negative than $-0.05 \mathrm{~V}_{\mathrm{SHE}}$. A single electron transfers from the semiconductor surface to a molecule for the molecular oxygen reduction, while the hydrogen production requires a stepwise reaction. Thus, the molecular oxygen reduction will take place preferentially.

In the present photocatalytic system, the bulk $\mathrm{WO}_{3}$ and $\mathrm{WO}_{3}$-QDs larger than $1.5 \mathrm{~nm}$ are not effective for photoreduction (Fig. 3b). The efficiency of the photoreduction of proton increased with expansion of the bandgap over $3.0 \mathrm{eV}$ by decreasing the size of $\mathrm{WO}_{3}$-QDs to $\sim 1.5 \mathrm{~nm}$. Moreover, the efficiency of 

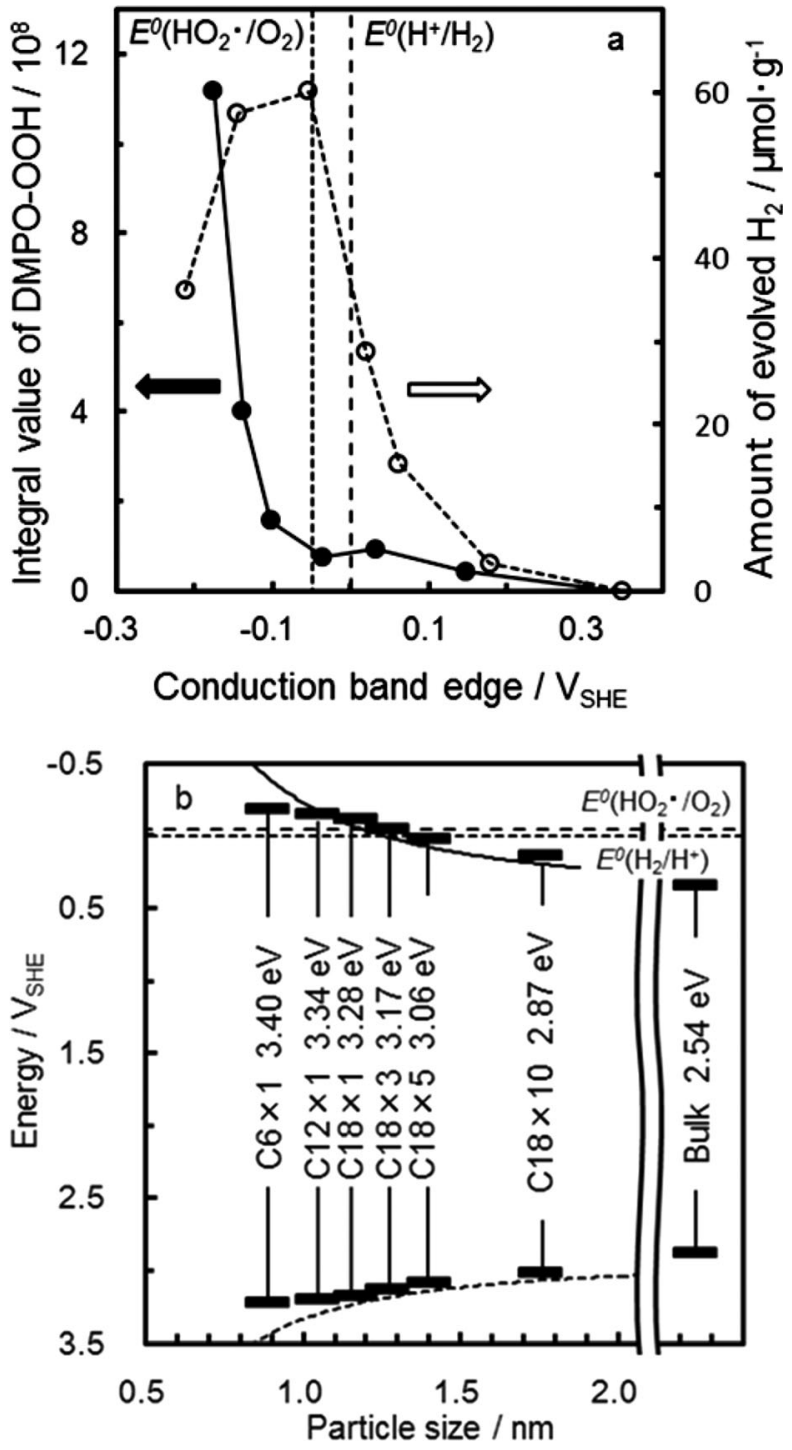

Fig. 3 (a) The relationship between the efficiency of photoreduction of proton (open circles) and molecular oxygen (closed circles) and the CBE potential of $\mathrm{WO}_{3}-\mathrm{QDs}$ and (b) band diagram of $\mathrm{WO}_{3}-\mathrm{QDs}$. The particle size of $\mathrm{WO}_{3}$-QDs was estimated from bandgap energy by using the Brus equation $\left(E_{\mathrm{g}}=2.87-3.30 \mathrm{eV}\right)$ and the approximation curve (dotted line for $E_{\mathrm{g}}=3.30-3.40 \mathrm{eV}$ ) in Fig. 1e.

the photoreduction of molecular oxygen steeply increased instead of hydrogen molecule evolution with an expansion of the bandgap over $3.3 \mathrm{eV}$ by decreasing the size of $\mathrm{WO}_{3}$-QDs to $\sim 1.0 \mathrm{~nm}$. Thus, we can tune the photocatalytic reduction by conduction band engineering of $\mathrm{WO}_{3}$-QDs via size control of $\mathrm{WO}_{3}$-QDs.

We revealed the correlation between the $\mathrm{CBE}$ of $\mathrm{WO}_{3}$-QDs and their photocatalytic activity without changing their composition by using the band engineering of the single metal oxide with the quantum size effect. The size of $\mathrm{WO}_{3}$-QDs was controlled by changing the pore size of the SMPSs and the amount of the precursor solution of the $\mathrm{WO}_{3}$-QDs impregnated into the SMPSs. The CBE of $\mathrm{WO}_{3}$-QDs was experimentally determined by surface complexing with phenol. We found that the efficiency of the photoreduction of proton and molecular oxygen increased steeply as the negative shift of the CBE from the reduction potential by decreasing the size of QDs in the range below $1.5 \mathrm{~nm}$. Moreover, tuning of the photocatalytic reduction, such as the formation of oxygen radicals and $\mathrm{H}_{2}$ evolution, was achieved by the control of the size of $\mathrm{WO}_{3}$-QDs. These results suggest the possibility of the design of an advance photocatalyst of a single semiconductor material by using its band engineering with the quantum size effect.

This work was partially supported by the Kato Foundation for Promotion of Science and Grant-in-Aid for Scientific Research (No. 22107010) on the innovative areas of "Fusion Materials: Creative Development of Materials and Exploration of Their Function through Molecular Control" (No. 2206) from the Ministry of Education, Culture, Sports, Science and Technology.

\section{Notes and references}

1 S. C. Roy, O. K. Varghese, M. Paulose and C. A. Grimes, ACS Nano, 2010, 4, 1259.

2 S. Sun, W. Wang, D. Li, L. Zhang and D. Jiang, ACS Catal., 2014, 4, 3498.

3 U. I. Gaya and A. H. Abdullah, J. Photochem. Photobiol., C, 2008, 9, 1.

4 S. N. Habisreutinger, L. S. Mende and J. K. Stolarczyk, Angew. Chem., Int. Ed., 2013, 52, 7372.

5 A. Fujishima and K. Honda, Nature, 1972, 238, 37.

6 Y. Nah, I. Paramasivam and P. Schmuki, ChemPhysChem, 2010, 11, 2698.

7 T. Inoue, A. Fujishima, S. Konishi and K. Honda, Nature, 1979, 277, 637.

8 I. Tsuji, H. Kato, H. Kobayashi and A. Kudo, J. Am. Chem. Soc., 2004, 126, 13406.

9 G. Liu, Z. Zhou and L. Guo, Chem. Phys. Lett., 2011, 509, 43.

10 Y. Wang and N. Herron, J. Phys. Chem., 1991, 95, 525.

11 P. V. Kamat, J. Phys. Chem. C, 2008, 112, 18737.

12 N. Satoh, T. Nakashima, K. Kamikura and K. Yamamoto, Nat. Nanotechnol., 2008, 3, 106.

13 H. Watanabe, K. Fujikata, Y. Oaki and H. Imai, Chem. Commun., 2013, 49, 8477.

14 L. Brus, J. Phys. Chem., 1986, 90, 2555.

15 P. E. Lippens and M. Lannoo, Phys. Rev. B: Condens. Matter Mater. Phys., 1989, 39, 10935.

16 E. L. Tae, S. H. Lee, J. K. Lee, S. S. Yoo, E. J. Kang and K. B. Yoon, J. Phys. Chem. B, 2005, 109, 22513.

17 M. T. Greiner, M. G. Helander, W. M. Tang, Z. B. Wang, J. Qiu and Z. H. Lu, Nat. Mater., 2012, 11, 76.

18 R. Konaka, E. Kasahara, W. C. Dunlap, Y. Yamamoto, K. C. Chien and M. Inoue, Free Radical Biol. Med., 1999, 27, 294.

19 J. Kim, C. W. Lee and W. Choi, Environ. Sci. Technol., 2010, 44, 6849 . 